\title{
Treatment of class III Malocclusion with maxillary expansion and face-mask therapy: A case report
}

\author{
Mansuri $\mathbf{M}^{1}$, Singh $\mathrm{VP}^{2}$ \\ B. P. Koirala Institute of Health Science, Dharan, Nepal
}

${ }^{1}$ Intern, College of Dental Surgery, B. P. Koirala Institute of Health Science, Dharan, Nepal.

${ }^{2}$ Associate Professor. Denartment of Orthodontics. College of Dental Surgerv. BPKIHS. Dharan.

\begin{abstract}
The developing class III malocclusion is one of the most challenging problems for the practicing orthodontist to manage clinically. True class III malocclusion is rare in our region as compared to Class II and Class I malocclusion. With adults, orthognathic surgery and dental camouflage is the viabletreatment option. A variety of treatment alternatives exists for patients in the developing stages of a Class III malocclusion. In the past much ofthetherapy has focused on restriction ofmandibulargrowth with chin cups and functional appliances.Thisis based on the traditional thought that developingClass III malocclusions were the result ofa prognathic mandible. Recently, however, there has been a growingawareness that the majority of patients with a developing Class III skeletal pattern exhibit a maxillary deficiency with a normal or only slightly prognathic mandible.
\end{abstract}

Therefore, considerable attention has been given to early treatment using maxillary protraction therapy. Using facemask therapy in conjunction with maxillary expansion has been shown in clinical reports to be a successful and predictable treatment option. Treatment should be carried out as early as possible with the aim to prevent it from becoming severe. A case treated with biphasic therapy - orthopaedic appliance followed by fixed orthodontic treatment is presented here.

Keywords: Class III malocclusion, Facemask, Maxillary expansion

\section{INRODUCTION}

Class III malocclusion are growth related developmental problems. If they are left untreated, they often become more severe.It should be corrected as soon as possible for the better and more stable outcomes. Developing true class III malocclusion tendencies in children may have an underlying skeletal or dental component ${ }^{1}, 2$. Pseudo Class III malocclusion is a habitual established crossbite of all anterior teeth without any skeletal discrepancy, resulting from functional forward positioning / shift of mandible on closure, so both should be differentiated before start of any treatment 
procedure $[1,2]$. When left untreated Pseudo class III may lead to development of true class III malocclusion. Class III malocclusions continue to be the mostchallenging to accurately diagnose and clinically manage [3]. The treatment should be carried out as early as possible with the aim of permitting normal growth [2].The main objective of early treatment is growth modulation during the most active stages of dental and craniofacial growth [4].

\section{CASE}

A 9 year old male patient reported to the department with the chief complaint of irregularly placed upper front teeth and forwardly placed lower jaw. Clinical examination revealed concave profile, anterior divergence, and upper and lower anterior crowding with posterior cross bite. Cephalometric analysis suggested skeletal class III malocclusion with deficient maxilla. The general health status of the patient was good with no significant medical history.

Records: study casts, OPG, cephalogram and photographs were taken for the evaluation and confirmation of the diagnosis.

General and Extra-oral examination: Mesomorphic body, Normal gait and posture, Mesoprosopic face with competent lips, Concave to almost straight profile.

Intraoral Examination: Soft tissues normal.

Erupted teeth Profile:

$$
\begin{array}{l|l}
654321 & 123456 \\
\hline 654321123456
\end{array}
$$

Maxillary arch: Asymmetrical with palataly placed lateral incisors, erupting canines and bilateral posterior crossbite.

Mandibular arch: Asymmetrical, completely overlapping the maxillary arch and rotated incisors with anterior crowding.

\section{Intra-occlusal examination:}

- Bilateral Angle's Class III molar relationship

- Bilateral Canine Class III relationship

- Bilateral posterior crossbite

- Edge to edge bite

\section{Diagnosis:}

- Skeletal class III malocclusion with retrognathic maxilla and normal mandible and bilateral posterior crossbite.

- Class III malocclusion with edge to edge bite.

\section{Treatment Objectives:}

Expanding and protracting the constricted maxilla achieving class I molar relationship and canine relationship with ideal overjet and overbite.

\section{Treatment plan:}

Biphasic therapy- RME to expand maxilla followed by facemask for maxillary protraction later fixed orthodontic treatment for final alignment.

\section{Treatment procedure:}


A bonded maxillary splint with hyrax (Fig. 1 and Fig. 2) was fabricated and hooks were placed in the canine premolar region above occlusal plane for attachment of elastics. First, RME was carried out using Timms protocol. After 3 weeks, Delaire face mask was given with this appliance to pull the maxilla forward (Fig. 3).
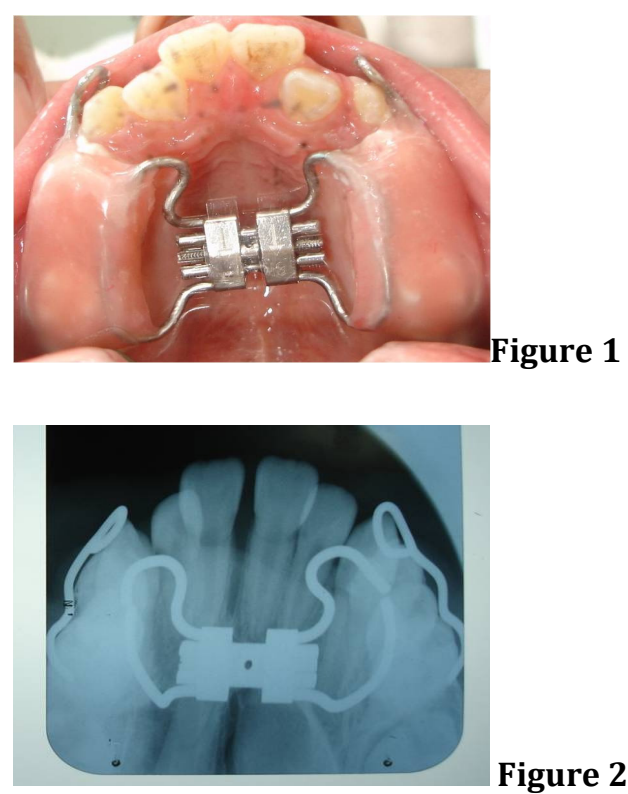

Figure 2

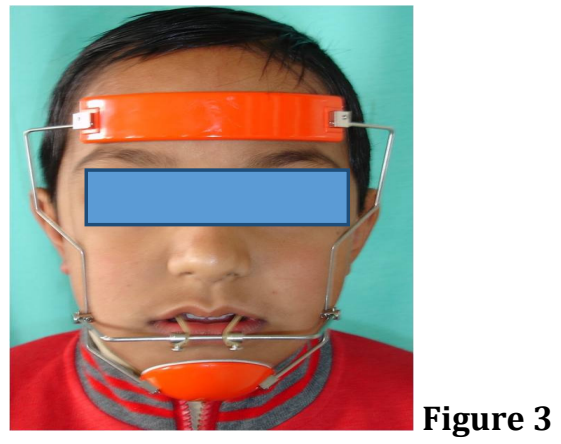

The total treatment time was 9 months and the appliance was held passively for 6 months for retention purpose.

Patient was shifted to fixed orthodontic treatment for: (Fig. 4 and Fig. 5)
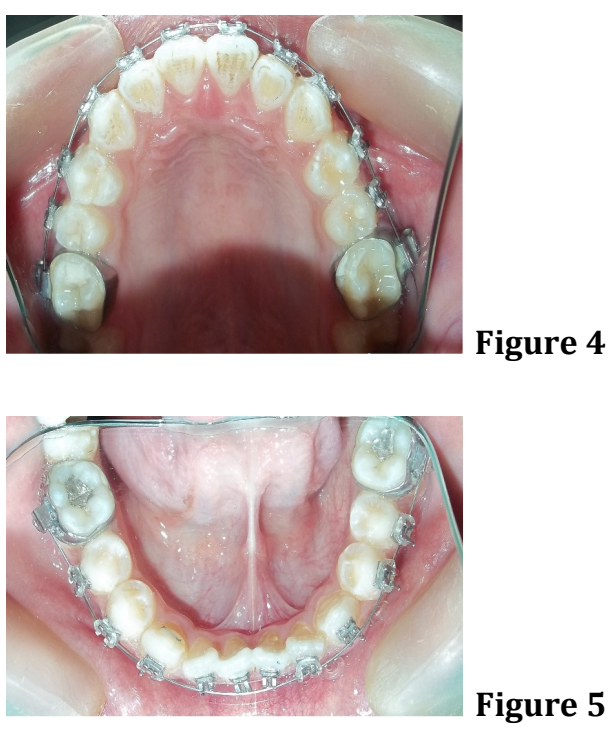

- Rotated incisors

- Finishing of occlusion

This phase took around 8 months.

Post-treatment: The post treatment Extraoral and Intra-oral findings can be seen clearly in figures

Table 1: Cephalometric analysis

\begin{tabular}{|c|c|c|}
\hline Parameters & $\begin{array}{c}\text { Pre- } \\
\text { treatment } \\
\text { value }\end{array}$ & $\begin{array}{c}\text { Post- } \\
\text { treatment } \\
\text { value }\end{array}$ \\
\hline SNA & $76.5^{\circ}$ & $82.5^{\circ}$ \\
\hline SNB & $79.5^{\circ}$ & $80.5^{\circ}$ \\
\hline ANB & $-3^{\circ}$ & $2^{\circ}$ \\
\hline $\begin{array}{c}\text { Go-Gn to SN } \\
\text { angle }\end{array}$ & $41^{\circ}$ & $41.5^{\circ}$ \\
\hline Y-axis & $56.5^{\circ}$ & $58^{\circ}$ \\
\hline Facial angle & $89^{\circ}$ & $91^{\circ}$ \\
\hline
\end{tabular}

Retention: The patient was given upper and lower fixed retainers along with FR III for retaining Skeletal class III correction. 
Figure 6: the Pre and post pictures in the study

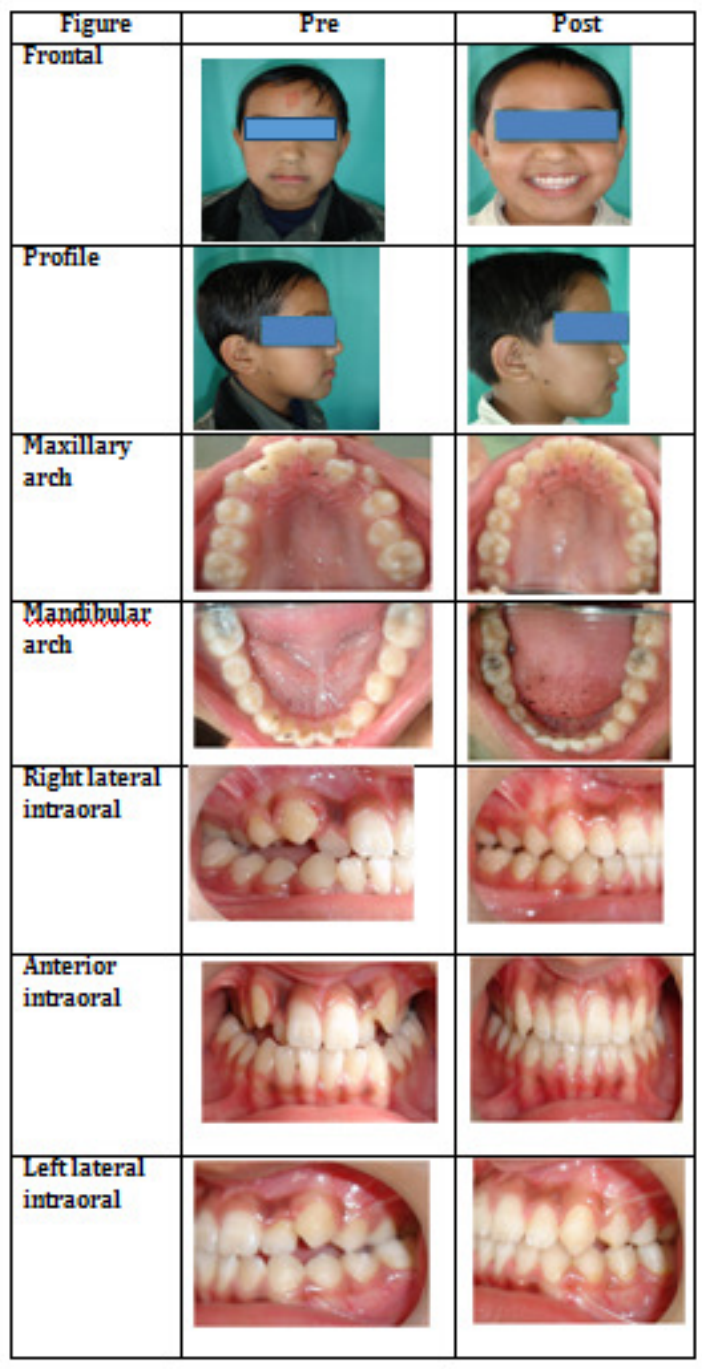

\section{DISCUSSION}

The developing class III malocclusion is one of the most challenging problems confronting the practicing orthodontists. If left untreated, the malocclusion tends to worsen and these patients will ultimately comprise a substantial percentage of patients seeking orthognathic surgery as adults [4, 5]. Thus, Class III malocclusion should be treated as early as possible for the better and stable outcomes. Early treatment by growth modulation is the choice of treatment. But this treatment takes a longer duration, so there are chances that patient will become unco-operative during the later stages of treatment [4].

In this case, we used biphasic therapy- RME (Rapid Maxillary Expansion) to expand maxilla followed by facemask therapy for maxillary protraction, and later fixed orthodontic treatment for final alignment. The facial growth pattern, skeletal pattern, age, appropriate appliance design and patient co-operation are all major factors in achieving the successful results of a stable occlusion ${ }^{4}$. In this case, there was a change of $6^{\circ}$ in the SNA angle after protraction of maxilla from $76.5^{\circ}$ to $82.5^{\circ}$ making the profile better (convex profile finally). Also, a change of $1^{\text {oin }}$ SNB angle from $79.5^{\circ}$ to $80.5^{\circ}$ due to normal mandibular growth during the treatment duration. Due to this, ANB angle also got improvement from $-3^{\circ}$ to $2^{\circ}$. There was also improvement in growth pattern due to change in growth axis from $56.5^{\circ}$ to $58^{\circ}$. Thus, there was a great achievement in the treatment outcome. Skeletal class III malocclusion should be corrected as soon as we recognize the initial sign as it can alter the normal craniofacial growth if left untreated [4].

\section{CONCLUSION}

Facemask and palatal expansion therapy is the choice for an effective and predictable approach for the treatment ofthe developing Class III malocclusion. Proper diagnosis and proper useofthe appliances and mechanics ensure the long-term results.The earlier the case presents to the clinic and is diagnosed, the simpler \& faster is treatment and earlier the treatment is carried out, the faster \& more stable are the results. 


\section{REFERENCES}

1. KukrejaManisha. $\mathrm{K}$ et al. Early Treatment of Class III Malocclusion. The Orthodontic Cyber Journal, January 2001. (http://orthocj.com/2011/01/earlytreatment-of-class-iii-malocclusion/)

2. Kapur A., Chawla H. S., Utreja A.; Early class III occlusal tendency in children and its selective management. J. Indian SocPedo Prevent Dent; Sept 2008; 26(3): 107-113.

3. Martin B. Epstein, Zoshua Z. Epstein and GarriTsibel; Management of the developing class III malocclusion with face mask therapy and palatal expansion. (http://www.scribd.com/doc/13496463/ Management-of-the-Developing-Class-IIIMalocclusion-with-Face-Mask-Therapyand-Palatal-Expansion)

4. MaheshwariS. and Gupta N. D.; Early treatment of Class III: a case report,J. Indian SocPedo Prevent Dent; December 2001; 19(4): 148151.

5. Graber T. M., Orthodontics: principle and Practice, Philadelphia WB Saunders Company, 1966. 